\title{
Some traffic features at freeway bottlenecks
}

\author{
Michael J. Cassidy*, Robert L. Bertini \\ Department of Civil and Environmental Engineering, Institute of Transportation Studies, University of California, \\ 109 McLaughlin Hall, Berkeley, CA 94720, USA
}

\begin{abstract}
Observations from two freeway bottlenecks in and near Toronto, Canada indicate that the average rate vehicles discharge from a queue can be $10 \%$ lower than the flow measured prior to the queue's formation. Absent any influences from downstream, the queue discharge flows exhibited nearly stationary patterns that alternated between higher and lower rates. These alternating flow patterns were especially evident at one of the two sites, although the feature occurred at both sites during periods that immediately followed the onset of upstream queueing; i.e. a queue's formation was always accompanied by a relatively low discharge rate followed later by a temporary surge in the discharge flow. When plotted cumulatively over time, however, the counts of discharging vehicles generally did not deviate by more than about 50 vehicles from a trend line of constant slope. Thus, the discharge flows are described as being 'nearly' constant; i.e. they varied (slightly) about a fixed rate. At each site, this average discharge rate exhibited little deviation from day to day. The present findings came by visually comparing transformed curves of cumulative vehicle arrival number vs time and cumulative occupancy vs time measured at neighboring loop detectors. This treatment of the data provided clear presentations of some important traffic features and this facilitated a detailed study of bottleneck flows. (C) 1998 Published by Elsevier Science Ltd. All rights reserved.
\end{abstract}

Keywords: Freeway capacity; Freeway bottleneck

\section{Introduction}

It is shown that the average rate freeway vehicles discharge from a queue can be $10 \%$ lower than the flow absent the queue. It is further shown that traffic in an active bottleneck ${ }^{* 1}$ exhibited 
nearly stationary patterns with discharge flows that alternated between higher and lower rates. In particular, a period of rather low discharge flow, followed by a period of relatively high flow, always accompanied the onset of upstream queues. These observations notwithstanding, the discharge flows might reasonably be described as 'nearly' constant, since the variations occurred about a fixed rate; i.e. the cumulative counts of discharging vehicles versus time seldom deviated by more than about 50 vehicles from a line of constant slope.

That the near-constant (i.e. average) discharge rate was less than the flow measured prior to queue formation was consistently observed at both bottlenecks studied herein. Furthermore, each bottleneck's average queue discharge rate exhibited only small day to day deviations; i.e. the differences observed in the average rates for any two days did not exceed $2 \%$. However, larger variations were sometimes observed in the daily measurements of the flows immediately before the onset of queueing and in the durations that these flows persisted. Likewise, the sequence of the very low and the very high discharge rates that immediately followed queue formations sometimes varied both in their magnitudes and in their observed durations.

\section{Background}

Some studies have reported that freeway capacity diminishes by an average of 3 or $4 \%$ following the formation of an upstream queue (Agyemang-Duah and Hall, 1991; Banks, 1990, 1991) while other studies report no such reductions (Newman, 1961; Persaud, 1986; Hall and Hall, 1990; Persaud and Hurdle, 1991). Oddly enough, two studies of the same bottleneck have claimed both extremes (Hall and Hall, 1990; Agyemang-Duah and Hall, 1991). We claim that findings from these earlier works were inconclusive due to the methods used to process the measured data; the diagnostics in most of these studies were time-series plots of flow, occupancy and/or average vehicle speed, and with these treatments, the data do not always reveal important traffic features. Further discussion of this is offered in Cassidy and Windover (1995).

Also relevant to our discussion, some of the literature hold that the 'gaps' commonly observed in scatterplots of traffic data (such as flow and occupancy) are evidence of capacity reductions caused by upstream queueing (Edie, 1961; Drake et al., 1967; Ceder and May, 1976; Payne, 1984; McShane and Roess, 1990; Agyemang-Duah and Hall, 1991; Hall et al., 1992). A recent study on the subject, however, showed that these gaps in the bivariate data may have been caused by traffic conditions emanating from downstream of the measurement locations (Cassidy, 1998).

Finally, a study of Japanese motorways reported that queue discharge rates decreased with time (Koshi et al., 1992). We observed, however, that a bottleneck's long-run discharge flow was nearly constant, except when influenced by traffic conditions from further downstream. An example of a downstream influence is illustrated later.

The present findings came by visually comparing transformed curves of cumulative vehicle arrival number vs time and cumulative occupancy vs time measured at neighboring (loop detector) locations. As shown in later sections, this treatment of the data revealed how some timedependent traffic features propagated over time and space. With these graphical presentations, we were able to identify the detectors that were located downstream of active bottlenecks and to study in detail the flows measured by these detectors. 


\section{The data}

The two freeway sites used in this study are the segments of the Queen Elizabeth Way (QEW) and the Gardiner Expressway illustrated in Fig. 1. Both sites are located in metropolitan Toronto, Canada and both have been featured in previous studies of capacity (Persaud, 1986; Hall and Hall, 1990; Agyemang-Duah and Hall, 1991; Persaud and Hurdle, 1991).

The loop detector stations for measuring traffic data are labeled in the figure as per the numbering strategies adopted by the Ontario Ministry of Transport and Metropolitan Toronto Transportation. The QEW detectors record counts, occupancies and (time) mean speeds in each lane over 30-s intervals and meters are deployed at the on-ramps upstream of the bottleneck. The detectors on the Gardiner Expressway collect the same measurements over intervals of 20-s duration and the expressway has no ramp metering.

The following two sections describe the bottleneck features observed (for single days) on the QEW and on the Gardiner Expressway. These observations were reproduced on additional days, as described in a later section (the dates of the data collection are shown in this later section as well). All measurements were made during days when the local weather bureau reported clear skies and no measurable precipitation.

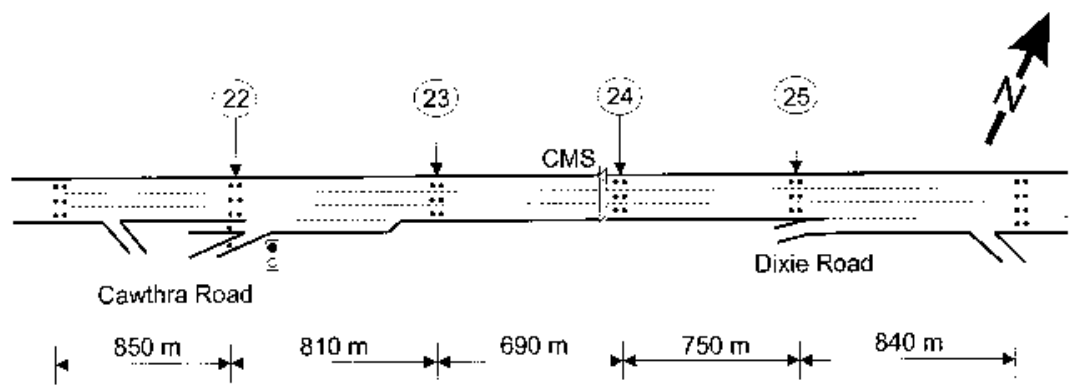

Queen Elizabeth Way

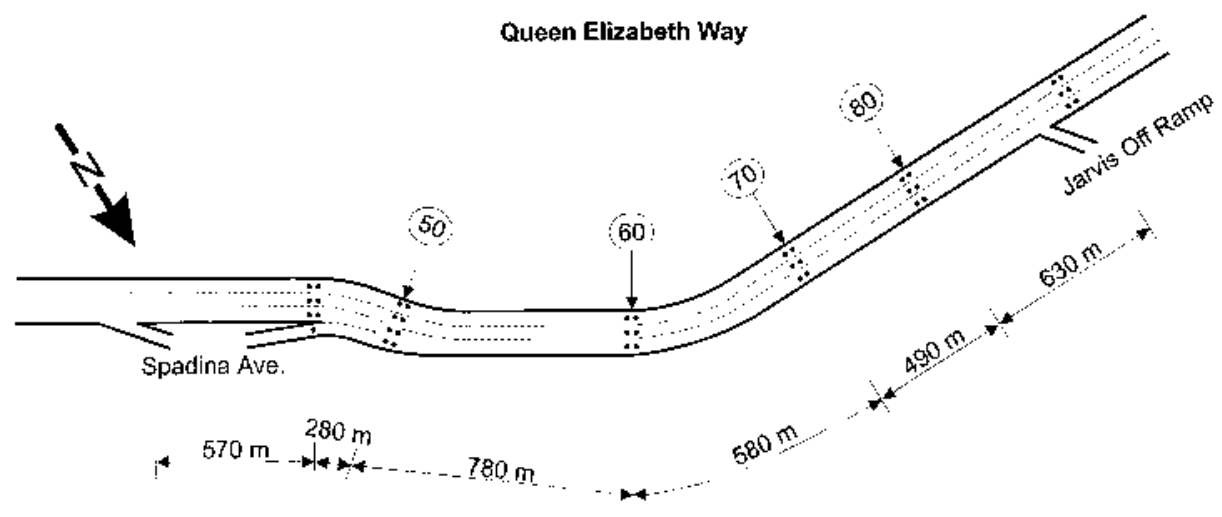

Gardiner Expressway

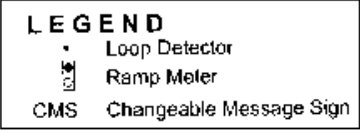

Fig. 1. Freeway sites. 


\section{Capacity features on the QEW}

Fig. 2 presents curves of $N(x, t)$ on the QEW, where

(a) $N(x, t)=$ the cumulative number of vehicles to pass (detector) location $\mathrm{x}$ by time $\mathrm{t}$, measured from the passage of some reference vehicle.

Here the $x$ refers to detector stations 22-25 (shown in Fig. 1). The vehicle counts used for the Fig. 2 curves were those measured in all three travel lanes and the curve for station 22 includes the counts at the Cawthra Road on-ramps (to maintain vehicle conservation between curves). The curves were adjusted as described below.

1. Piece-wise linear approximations to the $N(x, t)$ were constructed. If unaltered, the cumulative curves would have exhibited stair-step features because the detector measurements were recorded at hxed intervals. Our curves were constructed by taking linear interpolations through the outer edges of each stair-step; i.e., the resulting curve was the outer envelope of the stair-step curve. The slope of this curve at some time $t$ is the flow past $\mathrm{x}$ during the measurement interval containing the $t$.

2. The N-curves for stations 22, 23 and 24 were shifted horizontally to the right; each curve was translated by the average free-flow trip time from its respective $x$ to station 25, the downstream-most detector. Following these shifts, any vertical displacements between the curves were the excess vehicle accumulations due to traffic delays (Newell, 1993). Shifted along with the upstream curves were their corresponding time axes and separate axes for each $x$ are shown in Fig. 2.

3. Each N-curve was re-scaled by subtracting a background cumulative count, $q_{o} . t$, at all time $\mathrm{t}$, where $\mathrm{q}_{\mathrm{o}}$ is defined as the background flow and $t$ is the elapsed time from the passage of the reference vehicle. A suitable choice of $q_{0}$ promotes the visual identification of changing flows directly from the re-scaled curve, as shown in Cassidy and Windover (1995) and in Cassidy (1998). ${ }^{2}$

The $N$ at stations 24 and 25 remained approximately superimposed, indicating that traffic was flowing freely between these detectors. Some displacements in the Fig. 2 curves occurred as early as 6:24 and the reader may use a straightedge to verify that this early divergence in the curves was accompanied by a subtle rise in the flows at upstream stations 22 and 23. Following these (initial) curve displacements, more substantial delays and vehicle accumulations developed between stations 23 and 24 as a consequence of flow reductions at downstream stations 24 and 25; these reductions began at approximately 6:30:00 and 6:30:30, respectively. Temporary flow reductions such as these, which were eventually followed by higher rates, invariably occurred at the onset of upstream queueing; more evidence of this is shown later.

\footnotetext{
${ }^{2} \mathrm{~A}$ re-scaled cumulative curve displays the vertical displacements between the original curve and a chord (e.g. the line $\left.N=q_{o} . f\right)$. Some diagnoses made with the re-scaled curves are thus independent of the magnitude of the background rate reduction (i.e., the slope of the chord), including diagnoses based upon (1) the presence or absence of displacements between neighboring N-curves like those in Fig. 2 (2) the convexity or concavity of a curve; and (3) the vertical deviations between a re-scaled curve and some chord. The re-scaling trick can, however, alter certain attributes of a curve, such as the magnitudes of its local minima and maxima and the times they occur.
} 


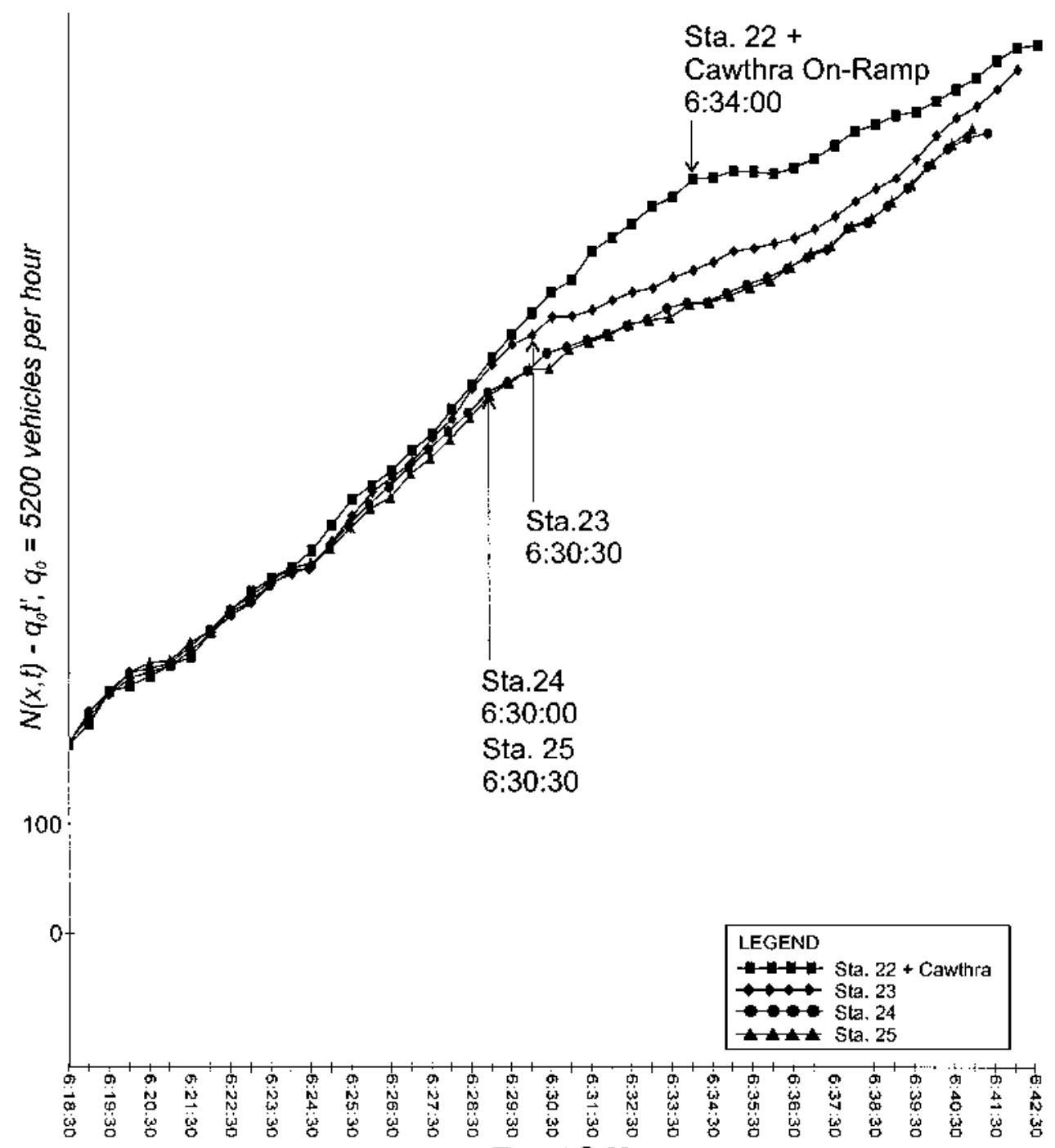

Time, $t @ 22$

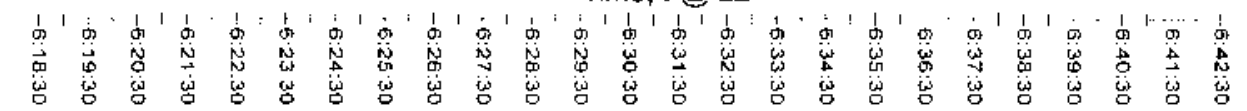
Time, t@23

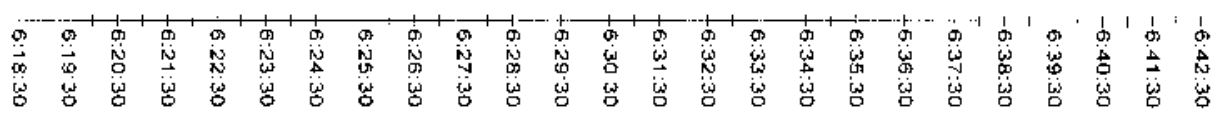

Time, t@ 24

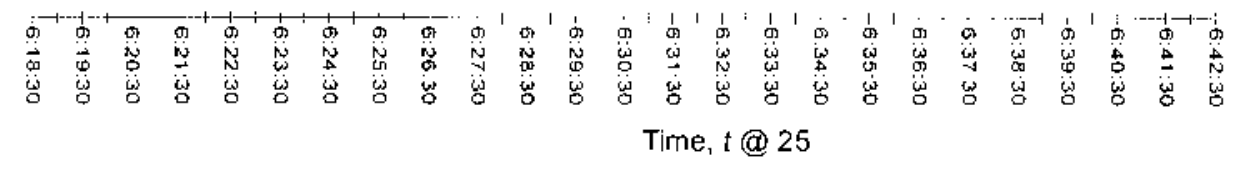

Fig. 2. Re-scaled ^^-curves, QEW. 
The divergence in the curve at station 23 from the one at station 22 (at 6:30:30) marks the arrival of a backward-moving queue at station 23. There was a pronounced flow reduction (at station 23) that accompanied this divergence, but the flow eventually returned to a higher rate. In previous work, temporary flow reductions such as this have been observed when the rear of a queue passes a measurement location (Cassidy and Windover, 1995). Fig. 2 also reveals that a flow reduction occurred at station 22 sometime around 6:34 and this indicates that the backwardmoving queue eventually propagated to this upstream station.

The evolution described above is corroborated by Fig. 3. Shown in this figure are curves of $T(x, t)-b_{o}(x) \cdot t^{\prime}$, where

(a) $\quad \mathrm{T}(\mathrm{x}, \mathrm{t})=$ the cumulative occupancy at station $\mathrm{x}$ to time $\mathrm{t}$, measured across all travel lanes; ${ }^{3}$

(b) $b_{0}(x)=$ the background rate used for re-scaling the $T$ (at a given $x$ ) to promote the visual identification of its time-dependent features (see footnote 2); the $t$ at each $\mathrm{x}$ is the elapsed time from the rather arbitrary starting point used for the curve.

Piece-wise linear approximations to the $T(x, t)$ were constructed so that the slope of the curve at some $t$ is the occupancy rate at detector station $x$ during the measurement interval containing that t. Each time axis in Fig. 3 corresponds to a unique $\mathrm{x}$, as labeled.

Referring to the lower portion of Fig. 3, the T-curves at stations 24 and 25 exhibit concave shapes. The periods marked by relatively low occupancy rates coincide rather closely with the flow reductions (at stations 24 and 25) that accompanied the onset of the upstream queue. The starting times for these downstream flow reductions were identified in Fig. 2 and these times are shown with arrows in the lower portion of Fig. 3 to serve as points of reference. The sequence of the changes shown in Fig. 2 and in the lower portion of Fig. 3 reveal the forward motion of a wave of lower flow and lower density, which we term an expansion wave. One would expect such a wave to emanate from a sudden (flow) restriction upstream.

In the upper portion of Fig. 3, the T-curves at stations 23 and 22 display convex shapes. At each of these two upstream stations, the time period marked by an increase in the occupancy rates coincides closely with the queue's arrival times as identified from Fig. 2; these latter times are shown (for the appropriate T-curve) in the top portion of Fig. 3. Reductions in flow (like those at stations 23 and 22 shown in Fig. 2) that are accompanied by increased occupancy rates (like those shown in the top portion of Fig. 3) are features we used to verify the arrival(s) of a backward-moving queue and additional examples of this are shown later.

In summary, the transformed $N$ and $T$ show that a forward-moving expansion wave, along with a backward-moving queue, emanated from between stations 23 and 24; i.e. the bottleneck was somewhere between these stations. We next show that station 25 remained downstream of an active bottleneck from the passage of the forward-moving expansion wave at 6:30:30 until the end of the morning peak. Station 25 was therefore a suitable measurement location for studying bottleneck flows. ${ }^{4}$

\footnotetext{
${ }^{3}$ Cumulative occupancy is measured in units of total time spent by vehicles atop the detectors at station $\mathrm{x}$ by time $\mathrm{t}$. Lin and Daganzo (1997) provide an extensive discussion of $T(x, t)$ curves for use in incident detection. Re-scaled T-curves have also been used to identify time periods of nearly stationary freeway traffic (Cassidy, 1998).

${ }^{4}$ Given the node conservation law, detector station 24 would have been an equally suitable location for measuring flows.
} 

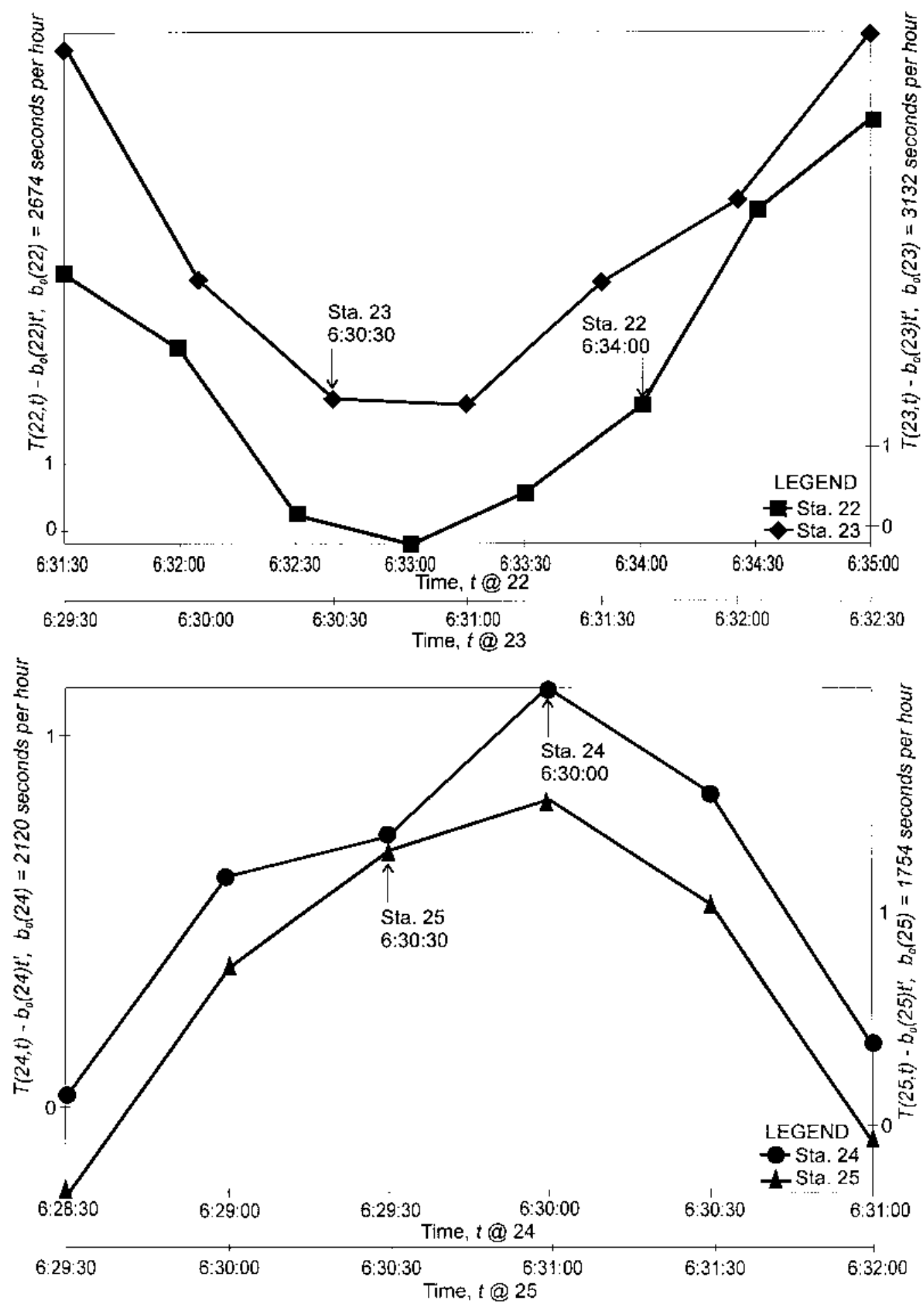

Fig. 3. Re-scaled T-curves, QEW. 
To verify the upstream queue's continued presence, Fig. 4 displays N-curves for station 22 (plus the Cawthra Road on-ramps) and for station 25. The Fig. 4 curves were constructed in the same way as those in Fig. 2, although the former span a longer duration. The displacements between the curves in Fig. 4 show that the queue (upstream of station 25) persisted until 7:54; it dissipated following the abrupt reduction in upstream flow measured at 7:49.

Finally, Fig. 5 presents re-scaled $\mathrm{N}$ - and T-curves at station 25. They reveal that the traffic conditions at this location were not influenced by downstream effects; i.e., the curves do not display any abrupt rises in the $T$ accompanied by reductions in the $\mathrm{N}$, features that would mark the arrival of a queue from downstream. To the contrary, both curves display remarkably similar features during the period of queue discharge. The correlations in the wiggles in the re-scaled $N$ and $\mathrm{T}$, and their quasi-linear trends (which are delineated by the vertical arrows in Fig. 5), reveal that the discharging vehicles exhibited sequences of nearly stationary traffic patterns with time

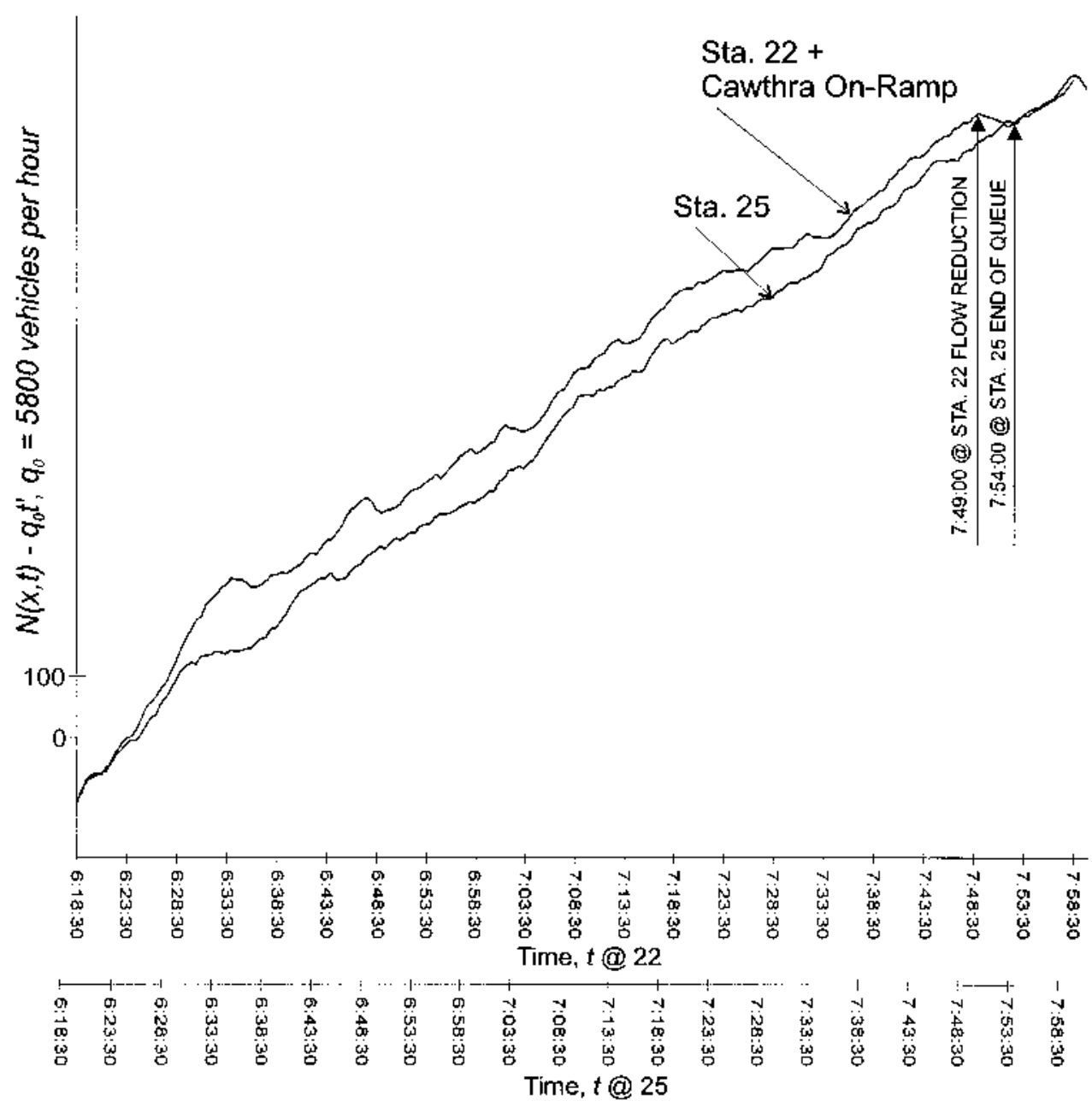

Fig. 4. Upstream and downstream N-curves, QEW. 


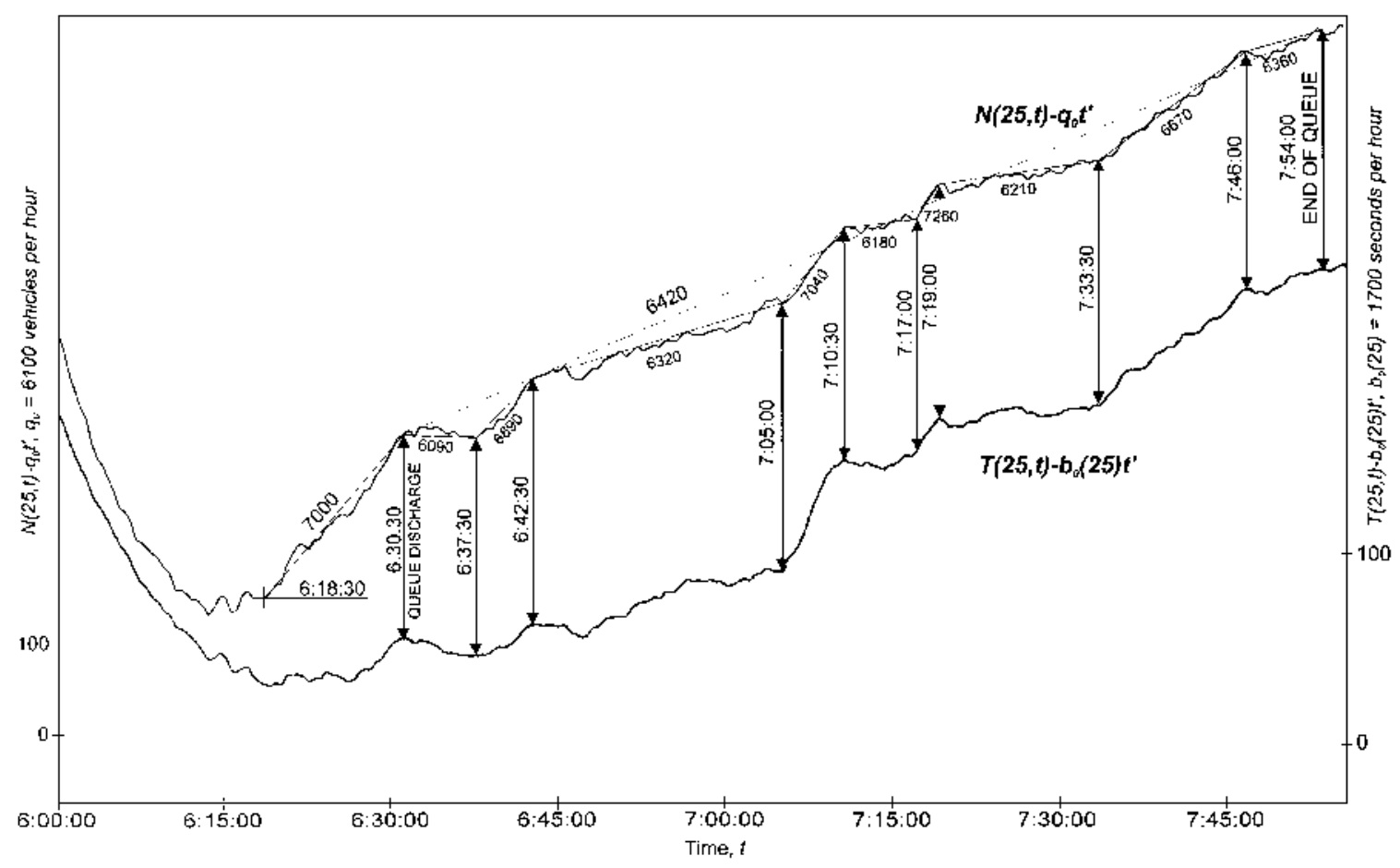

Fig. 5. Re-scaled $N$ and $T$, station 25 of the QEW.

dependent flows. ${ }^{5}$ Each near-stationary period is further highlighted in Fig. 5 by means of linear approximations superimposed on the $N$ and by labels which designate the hourly discharge flows measured during each of these periods.

The flow pattern in this figure might be described as sequences of surges followed by reductions, beginning with the high flow that prevailed prior to the onset of queue discharge. This high rate of 7000 vehicles per hour (vph), which is also labeled in the figure, prevailed for 12 minutes and ended with the arrival of the forward expansion wave. We note that the expansion wave carried a discharge flow of $6090 \mathrm{vph}$, the lowest rate observed during the peak, and that this rate persisted for $7 \mathrm{~min}$. Following this was a 5-min period marked by a high discharge rate of 6890 vph, which we refer to as the recovery discharge flow. Fig. 5 also shows that, on occasion, discharging vehicles exhibited flows that approached, and even exceeded, $7000 \mathrm{vph}$, the rate observed prior to upstream queueing.

The variation in the queue discharge flows occurred about a constant rate and this fixed trend is shown as a dashed line in Fig. 5. While the bottleneck was active, the $N$ never deviated by more than about 40 vehicles from the trend line. As a final note, the average queue discharge rate of $6420 \mathrm{vph}$ (which is labeled on the figure) is $8 \%$ lower than the flow (of $7000 \mathrm{vph}$ ) that prevailed prior to the upstream queue's formation.

\footnotetext{
${ }^{5}$ The intervals delineated by vertical arrows in Fig. 5 mark periods whereby the re-scaled $N$ and $T$ exhibited only small deviations from chords. Each of these intervals was characterized by some nearly constant flow and a nearly uniform vehicle speed; the rationale behind this diagnostic is explained in Cassidy (1998).
} 


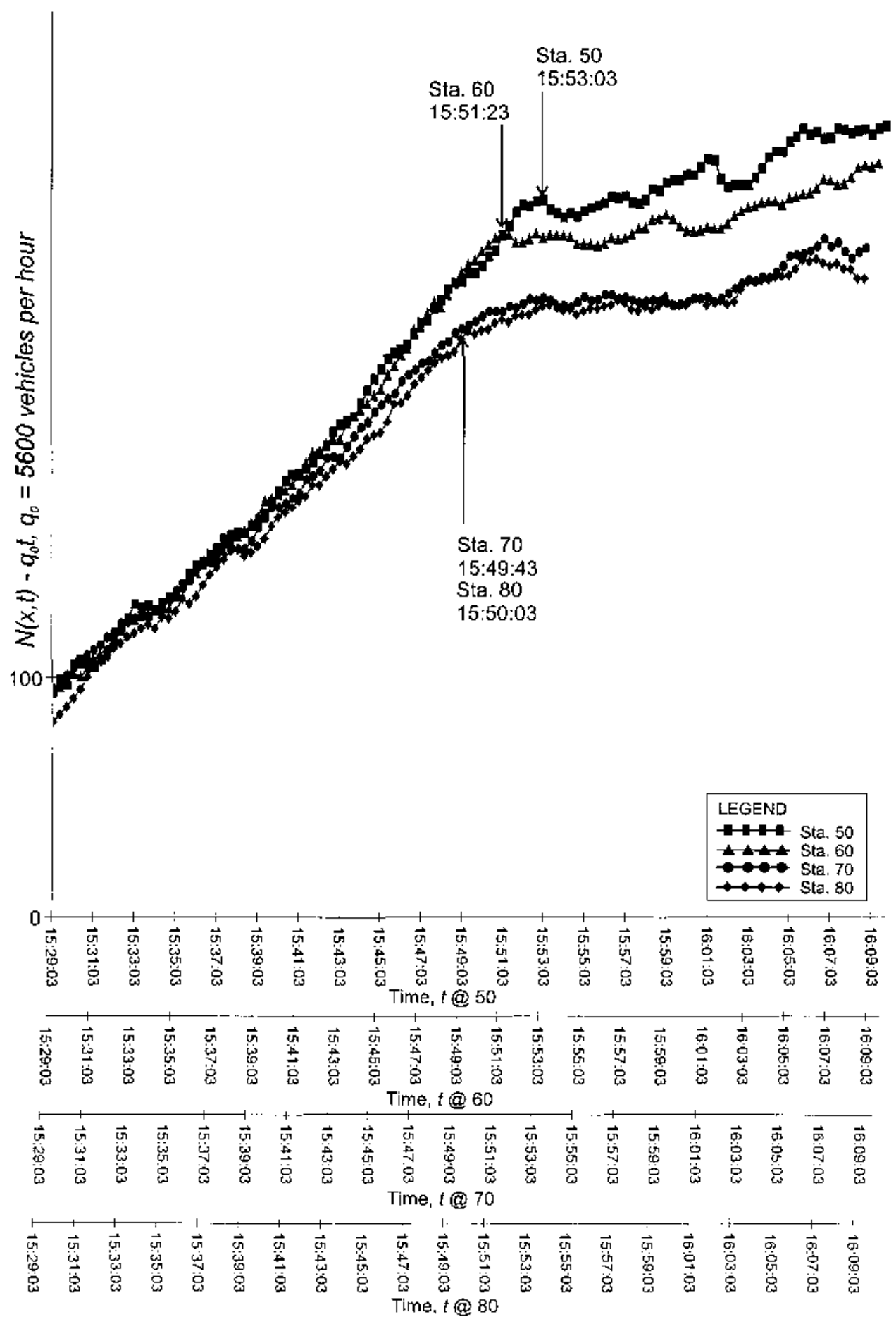

Fig. 6. Re-scaled ^^-curves, Gardiner Expressway. 

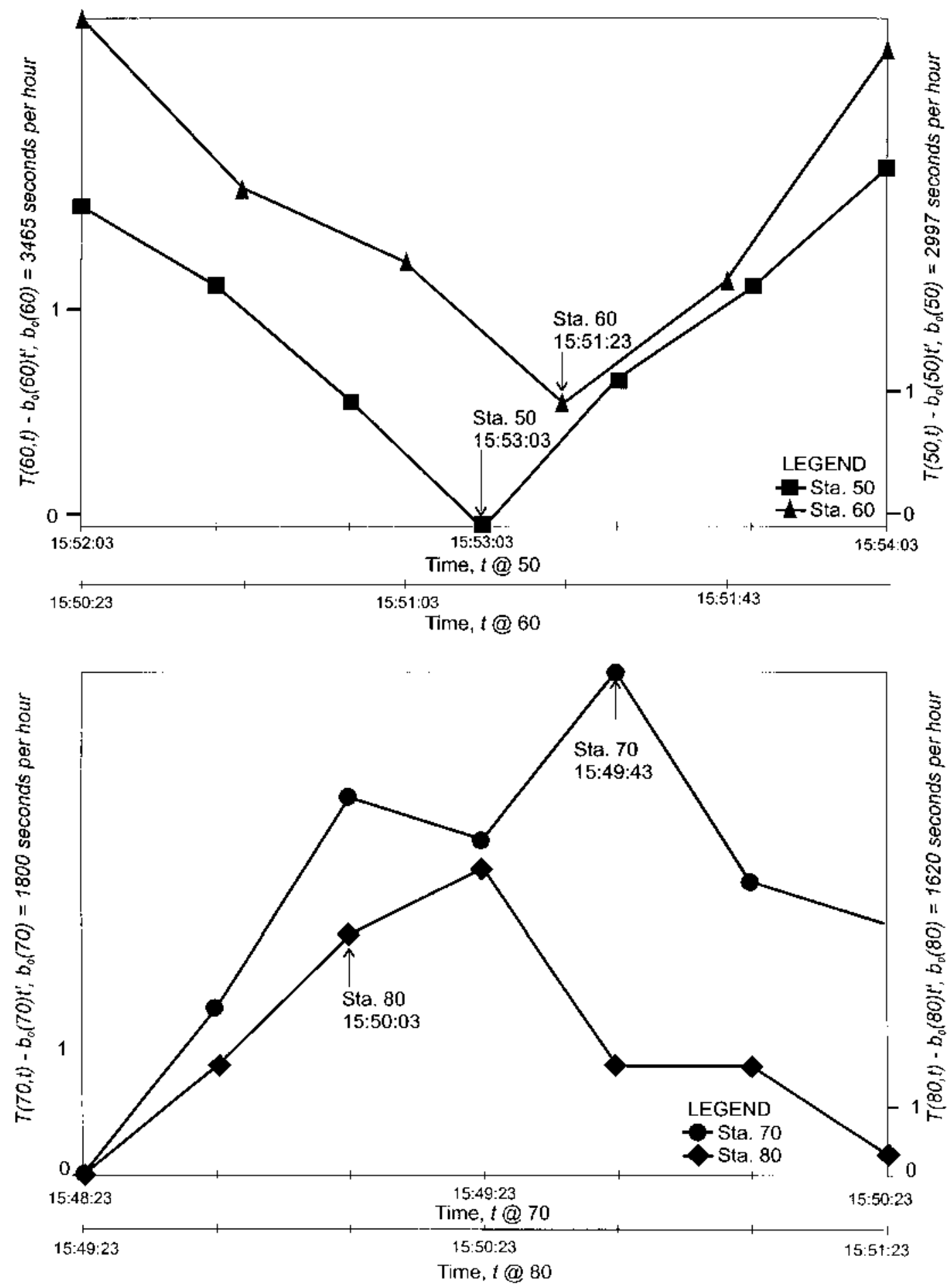

Fig. 7. Re-scaled T-curves, Gardiner Expressway. 


\section{Capacity features on the Gardiner Expressway}

Fig. 6 presents the transformed $N(x, t)$ curves for our segment of the Gardiner Expressway; these curves were constructed as previously described and here $\mathrm{x}$ refers to detector stations 50-80 (as illustrated in Fig. 1). These curves reveal that the queue formed between stations 60 and 70. Similar to the QEW curves in Fig. 2, the Fig. 6 curves begin to diverge when flows increase at upstream stations 50 and 60. Shortly thereafter, flow reductions occurred at downstream station 70 (at 15:49:43) and station 80 (at 15:50:03); these reductions were temporary as shown later in Fig. 10. The queue's arrival at station 60 (at 15:51:23) is signaled by a (temporary) flow reduction at this station and this causes a divergence in curves 50 and 60 .

The queue's evolution is corroborated via the re-scaled T-curves shown in Fig. 7; the times annotated on this figure correspond to the starting times of the flow reductions (as observed from Fig. 6). By examining the lower portion of Fig. 7, the T-curves for downstream stations 70 and

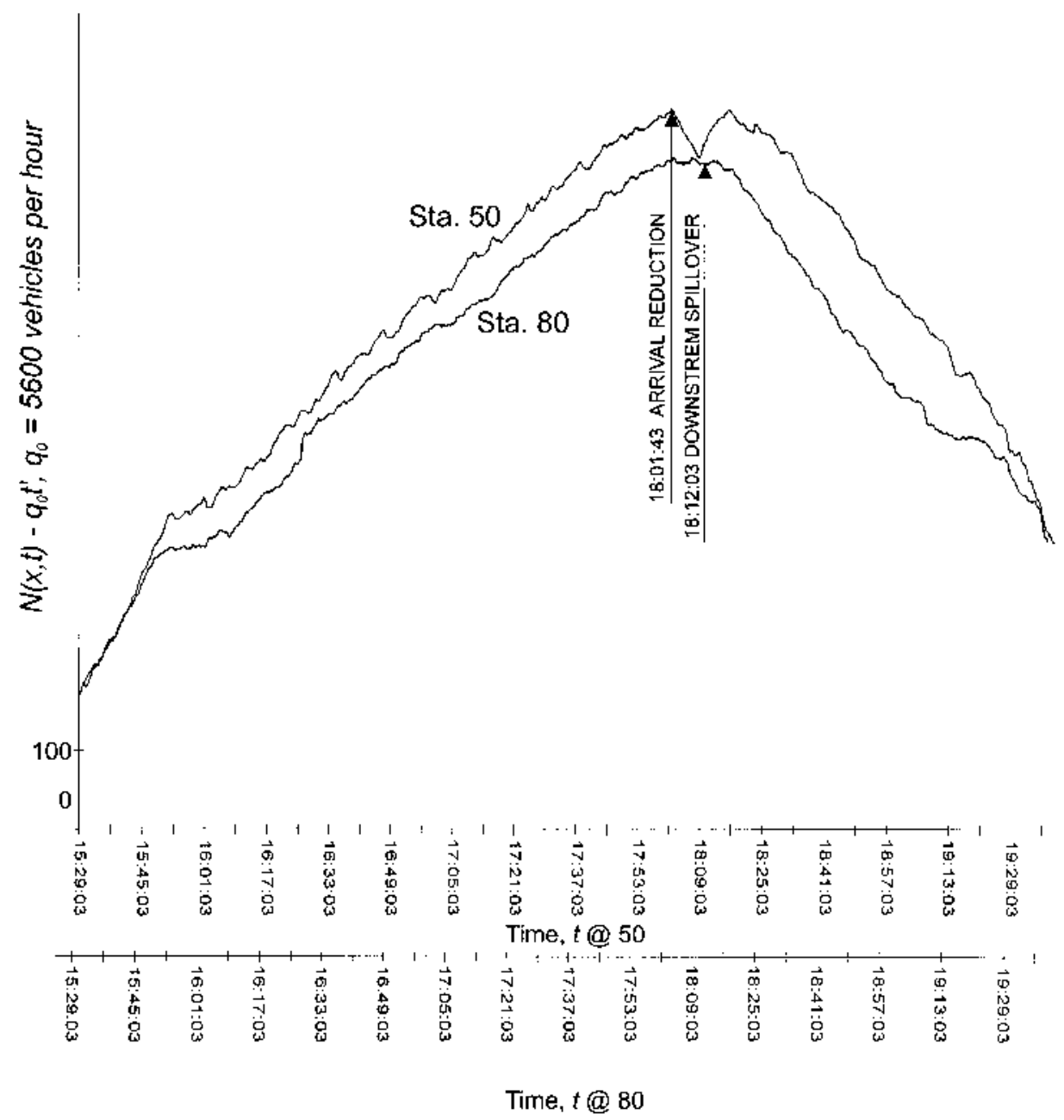

Fig. 8. Upstream and downstream N-curves, Gardiner Expressway. 
80, together with the corresponding N-curves in Fig. 6, it is evident that a forward-moving expansion wave propagated past these two stations in sequence. Viewing the upper portion of Fig. 7 along with Fig. 6 reveals that the queue emanated from downstream of station 60 and propagated in the upstream direction.

Having established that a bottleneck was between stations 60 and 70, we next show that this bottleneck was de-activated when a queue from downstream spilled-over some time later. As a consequence, station 80 was a suitable location for measuring bottleneck flows for only a portion of the peak period.

Fig. 8 displays N-curves at station 80 and at station 50. These curves, which span a period of over $4 \mathrm{~h}$, were constructed in our usual way. As an aside, the sharp drop in flow past station 50 at 18:01:43, and the flow increase shortly thereafter, emanated from upstream (as revealed by $\mathrm{N}$ and T- curves not shown here). More to the point, the slope of the curve at station 80 drops dramatically at around 18:12 and a similar slope reduction is displayed by the curve at station 50 shortly thereafter. Even after these flow reductions, the queue between stations 50 and 80 persisted, as revealed by the displacements in the curves. These curve attributes indicate that a queue from downstream arrived to station 80 (at around 18:12) and propagated further upstream.

This queue's arrival at station 80 is verified in Fig. 9, which presents curves of re-scaled $N$ and $T$ at this

station. The divergence in the Fig. 9 curves at 18:12:03 marks the arrival of the queue from downstream.

Finally, Fig. 10 shows that station 80 measured discharge flows from an active bottleneck from the arrival of the forward expansion wave at 15:50:03 until the queue arrived from downstream at 18:12:03. During this period, the Fig. 10 curves do not display any rises in the $T$ accompanied by reductions in the $\mathrm{N}$. To the contrary, the period is marked by near-stationary traffic with an alternating pattern of higher and lower discharge rates. As on the QEW, a dramatic flow reduction occurred at the onset of the upstream queue; in this instance, the low discharge rate of 5740 vph persisted for almost 20 min. Following this period, we observe a recovery discharge rate of $6150 \mathrm{vph}$, followed by a short-lived surge of $7920 \mathrm{vph}$. After this surge, the discharge flow settled to a nearly constant rate that prevailed until about 18:04:03; i.e. both the re-scaled $N$ and $T$ exhibit a consistent trend between 16:29:03 and 18:04:03.

The average discharge rate of $5950 \mathrm{vph}$ is shown with the dashed line in Fig. 10. Also shown is the flow of $6490 \mathrm{vph}$ that prevailed for $21 \mathrm{~min}$ before the queue formed upstream. Thus, the average queue discharge rate was about $8 \%$ lower than the maximum flow in the absence of the queue.

It is clear from Fig. 10 (and from Fig. 8) that after 18:12:03, the flow at station 80 was suppressed by the queue from downstream. We found that in the presence of an upstream queue, the re-scaled $N$ exhibited long-run diminutions from a nearly-constant trend only when traffic was influenced by downstream conditions; the queue arriving at station 80 at 18:12:03 is an example of a downstream influence. While a bottleneck remained active, however, its long-run discharge rate did not decrease with time.

We do observe a very low flow from 18:04:03 to 18:12:03, labeled as a 'flow drop' in Fig. 10. Ongoing research is investigating whether this 8-minute period marked a transition from the discharge flow in an active bottleneck to the traffic conditions in the downstream queue. ${ }^{6}$

\footnotetext{
${ }^{6}$ For now, we have included the 'flow drop' in our measurement of the average discharge flow. This inclusion lowered the average rate by only $30 \mathrm{vph}$, but did cause the re-scaled $\mathrm{N}$-curve to deviate by an unusually large amount from the trend line (shown as a dashed line in Fig. 10).
} 


\section{Reproducing the observations}

The analyses described in the previous two sections were repeated using data taken during two additional mornings on the QEW and during two additional afternoons on the Gardiner Expressway. In this section, we show that the general traffic features reported above were reproduced at each site for the additional days studied, although some variations were observed. Table 1 summarizes the six observations. For each site, the days used for data collection are labeled 'Day i,' $\mathrm{z}=1,2,3$, and the observations presented in the previous two sections are shown as 'Day 1 ' in the table.

We begin by describing the variations observed in some of the bottleneck features. To this end, Table 1 shows that on Day 3 at the Gardiner Expressway, the flow immediately prior to the upstream queue formation deviated from the other two observation days of this site. At both sites, the observed durations of these high flows prior to queueing varied considerably. Likewise, the magnitudes and the durations of the discharge flows that followed the onset of queueing

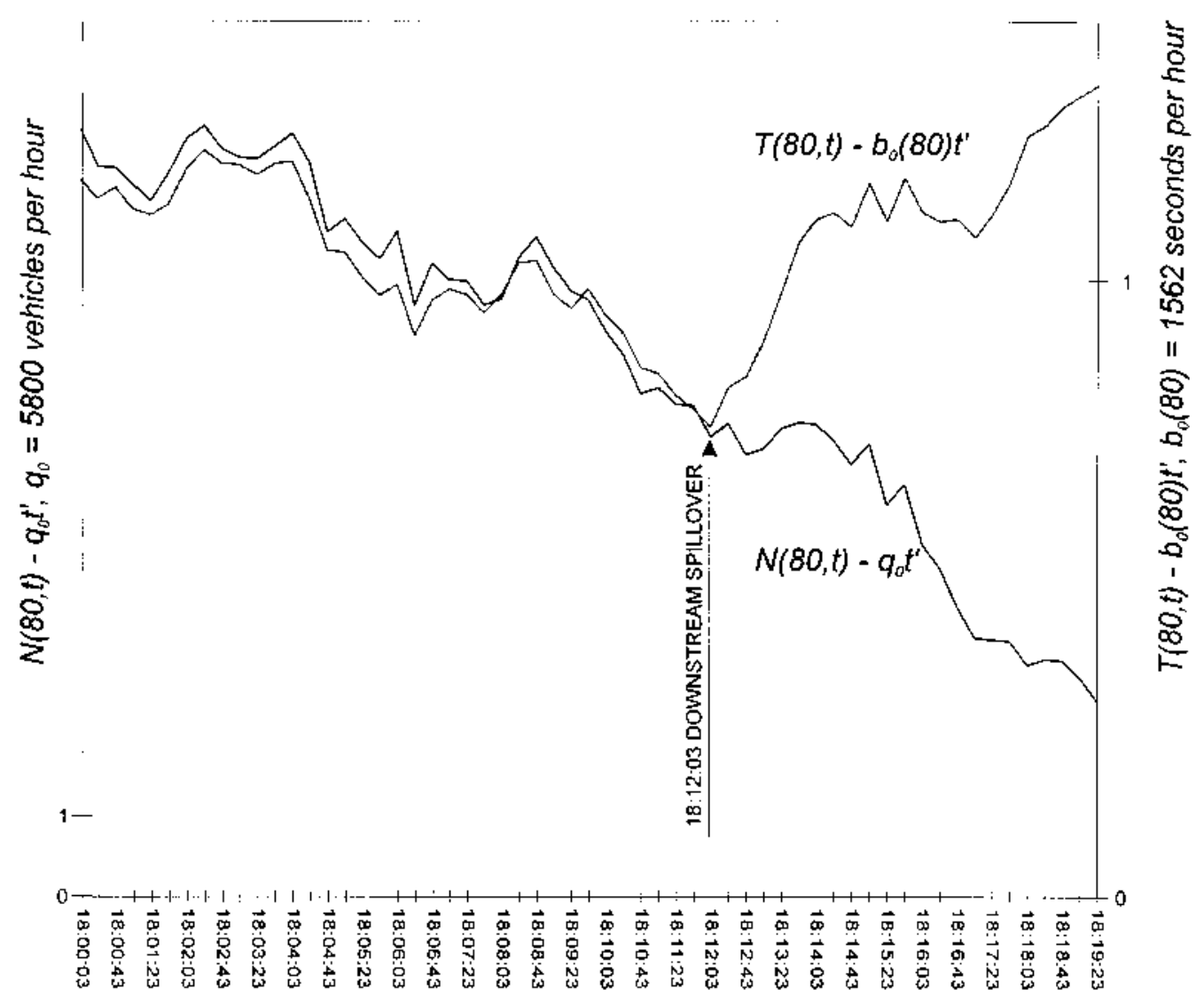

Time, $t @ 80$

Fig. 9. Re-scaled $N$ and $T$ showing the Queue’s arrival, Gardiner Expressway. 


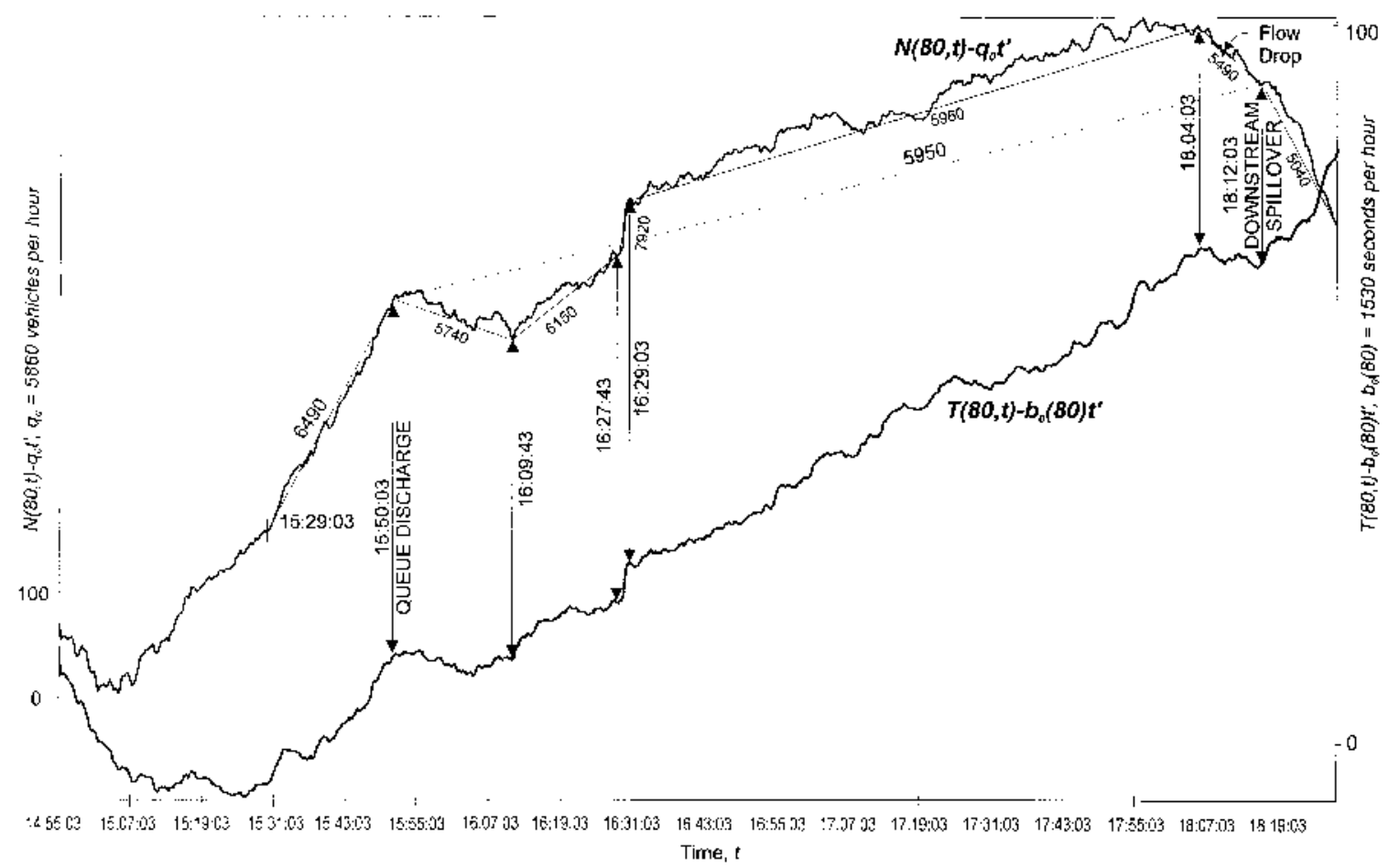

Fig. 10. Re-scaled $N$ and $T$, station 80 of the (jardiner Expressway.

(including the recovery discharge rates) exhibited daily variations at both sites. During two days at the Gardiner Expressway, the recovery discharge flows consisted of two high rates in sequence (as in Fig. 10); each of these rates and their respective durations are included in Table 1.

Table 1 also shows that some bottleneck features were reproducible from day to day and from site to site. For example, that the onset of queueing was accompanied by a low discharge flow followed by a higher recovery rate (or a sequence of two high rates) was a reproducible feature of both sites on each day observed. Likewise, Table 1 shows that the average queue discharge rates were consistent in that the day to day differences at a given site never exceeded $2 \%$. We further note that these discharge rates always persisted for long periods.

Also consistent at both sites was the daily observation that the flow immediately prior to queue formation was substantially larger than the average discharge rate. On five of the six days, the difference between these rates was at least $8 \%$, and in one instance, a difference of $10 \%$ was observed. The day with a difference of only 4\% (Day 3 at the Gardiner Expressway) was in mid summer, while all other observations were made in winter and spring. To what extent 'seasonal effects' influence the features of bottleneck capacity is part of ongoing study.

Although not shown in Table 1, the bottleneck location on each freeway site was consistent each day, at least within the resolution provided by the loop detectors. The QEW bottleneck always occurred between stations 23 and 24, while the bottleneck on the Gardiner Expressway always formed between stations 60 and 70; these locations are about a kilometer or more downstream of on-ramps (as shown in Fig. 1). 
Table 1

Summary of measured traffic features

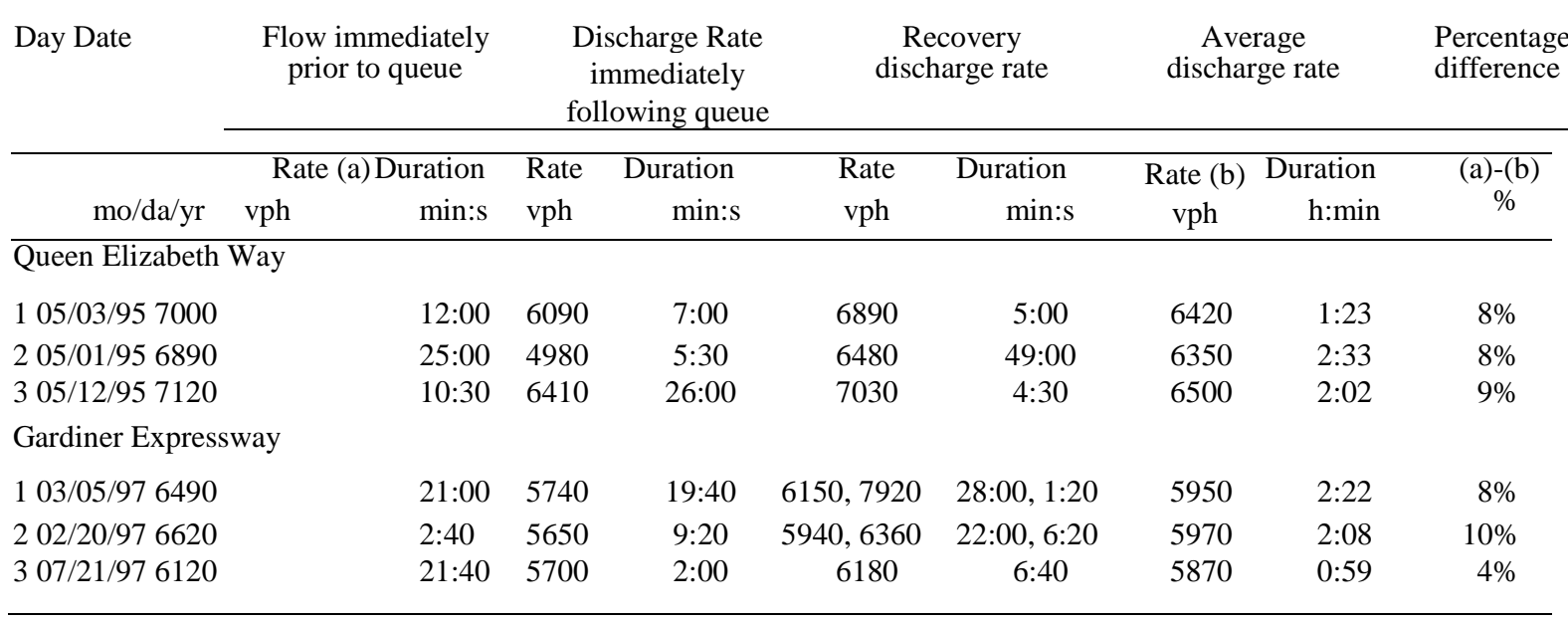

\section{Some final remarks}

We have observed that (1) flow can drop substantially following the formation of an upstream queue; (2) the discharge flows in active bottlenecks exhibit near-stationary patterns that (slowly) alternate about a constant rate; (3) a bottleneck's long-run discharge rates are consistent from day to day, while other flow features exhibit daily variation; and (4) bottlenecks occur at fixed (i.e. reproducible) locations. Our inclination is to view the long-run queue discharge flow as the bottleneck capacity given that these near-constant rates were sustained for prolonged periods and that they were replicated (approximately) each day. In contrast, the high flows measured prior to upstream queue formations persisted for short durations (compared to the peak periods) and these durations varied from day to day. This suggests that these flows were unstable and we are therefore reluctant to view the high flow prior to queueing as capacity.

The onset of upstream queueing was always accompanied by an especially low discharge flow followed by a recovery rate and these are the effects of driver behavior we do not yet understand. Although we find this an interesting as well as puzzling feature, it may be of little practical importance from the standpoint of modeling queue evolution; given that a cumulative curve of discharging vehicles never deviated much from a straight line, the discharge flow patterns that immediately followed a queue's formation would have little influence on predicting the evolution of the rear of the queue. Thus, the alternating pattern observed in the discharge flows does not invalidate the hydrodynamic theory of traffic as proposed byLighthill and Whitham (1955).

Further, the driver behavior that caused the high flows to break-down and to form queues, and the reasons these queues always formed about a kilometer or more downstream of on-ramps, are two more examples of the bottleneck features yet to be understood. In fact, all the findings presented here are effects, and research to understand their cause(s) is ongoing. We believe that the diagnostic methods described here can be refined, and perhaps augmented with additional measurements, to uncover explanations for at least some of the present findings. At present, for example, we are examining of Wcurves made from counts in individual travel lanes. We also 
intend to augment cumulative curves with vehicle trajectories extracted (over short freeway segments) from video using machine vision technology (Coifman, 1997). By examining the trajectories between detectors together with the $\mathrm{N}$-curves, including the $\mathrm{N}$-curves of individual lanes, we hope to obtain a better understanding of driver behavior at bottlenecks. With this understanding, we intend to explore issues such as the extent to which periods of relatively low discharge flows might be eliminated via control methods like ramp metering.

Finally, to what extent freeway bottleneck features in Toronto, Canada are similar to those in other regions of North America is not known. Thus, planned work includes the examination of bottlenecks on freeways in the United States.

\section{Acknowledgements}

The authors are indebted to Msrs. David Tsui and Mark Fox, Ontario Ministry of Transportation, and to Mr. Dave Nesbitt, Metropolitan Toronto Transportation, for providing the data used in this study. We also thank the (anonymous) referees for their helpful comments.

\section{References}

Agyemang-Duah, K., Hall, F.L., 1991. Some issues regarding the numerical value of capacity. Proceedings of the International Symposium of Highway Capacity. A.A. Balkema press, Germany, 1-15.

Banks, J.H., 1990. Flow processes at a freeway bottleneck. Transportation Research Record 1287, 20-28.

Banks, J.H., 1991. Two-capacity phenomenon at freeway bottlenecks: a basis for ramp metering?. Transportation Research Record 1320, 83-90.

Cassidy, M.J., Windover, J.R., 1995. Methodology for assessing the dynamics of freeway traffic flow. Transportation Research Record 1484, 73-79.

Cassidy, M.J., 1998. Bivariate relations in nearly stationary highway traffic. Transportation Research 32B, 49-59.

Ceder, A., May, A.D., 1976. Further evaluation of single- and two-regime traffic flow models. Transportation Research Record 567, 1-15.

Coifman, B., 1997. Time space diagrams for thirteen shock waves. California PATH working paper, UCB-ITS-PWP97-1, University of California, Berkeley, USA.

Daganzo, C.F., 1997. Fundamentals of Transportation and Traffic Operations. Elsevier Science Inc, New York, pp. 133-135, 259.

Drake, J.S., Schofer, J.L., May, A.D., 1967. A statistical analysis of speed density hypothesis. Highway Research Record 154, 53-87.

Edie, L.C., 1961. Car following and steady-state theory for non-congested traffic. Operations Research 9, 61-76.

Hall, F.L., Hall, L.M., 1990. Capacity and speed-flow analysis of the Queen Elizabeth Way in Ontario. Transportation Research Record 1287, 108-118.

Hall, F.L., Hurdle, V.F., Banks, J.H., 1992. Synthesis of recent work on the nature of speed-flow and flow-occupancy (or density) relationships on freeways. Transportation Research Record 1365, 12-18.

Koshi, M., Kuwahara, M., Akahane, H., 1992. Capacity of sags and tunnels in Japanese motorways. ITE Journal, May, 17-22.

Lighthill, M.J., Whitham, G.B., 1955. On kinematic waves II: a theory of traffic flow on long crowded roads. Proceedings of the Royal Society A229, 317-345.

Lin, W. F., Daganzo, C. E., 1997. A simple detection scheme for delay-inducing freeway incidents. Transportation Research 31A, 141-155.

McShane, W.R., Roess, R.P., 1990. Traffic Engineering, Prentice Hall, New Jersey, p. 290. 
Cassidy, Michael J.; Bertini, Robert L. Transportation Research Part B 33(1) 25-42. 1999.

Newell, G.F., 1993. Simplified theory of kinematic waves in highway traffic I: general theory. Transportation Research 27B, 281-287.

Newman, L., 1961. Study of traffic capacity and delay at the merge of the North Sacramento and Elvas freeways. report, California Division of Highways, USA.

Payne, H., 1984. Discontinuity in equilibrium traffic flow. Transportation Research Record 971, 140-146.

Persaud, B.N., 1986. Study of a freeway bottleneck to explore some unresolved traffic flow issues. PhD Dissertation. University of Toronto, Canada.

Persaud, B.N., Hurdle, V.F., 1991. Freeway capacity: definition and measurement issues. Proceedings of the International Symposium of Highway Capacity, A.A. Balkema press, Germany, pp. 289-307. 